\title{
Effect of Lipid Charge on Membrane Immersion of Cytochrome P450 3A4
}

\author{
Supporting Information
}

\author{
Veronika Navrátilová, Markéta Paloncýová, Karel Berka, Michal Otyepka* \\ Regional Centre of Advanced Technologies and Materials, Department of Physical \\ Chemistry, Faculty of Science, Palacký University Olomouc, tř. 17. listopadu 12, 771 46, \\ Olomouc, Czech Republic
}

Table S1. Number of ions and water molecules in simulations of CYP3A4 of various lipid bilayers.

\begin{tabular}{c|c|c|c} 
Lipid & WAT & $\mathrm{NA}^{+}$ & $\mathrm{CL}^{-}$ \\
\hline DOPC & 16967 & 46 & 47 \\
DOPE & 15801 & 41 & 42 \\
DOPG & 16730 & 274 & 45 \\
DOPS & 14528 & 293 & 60
\end{tabular}




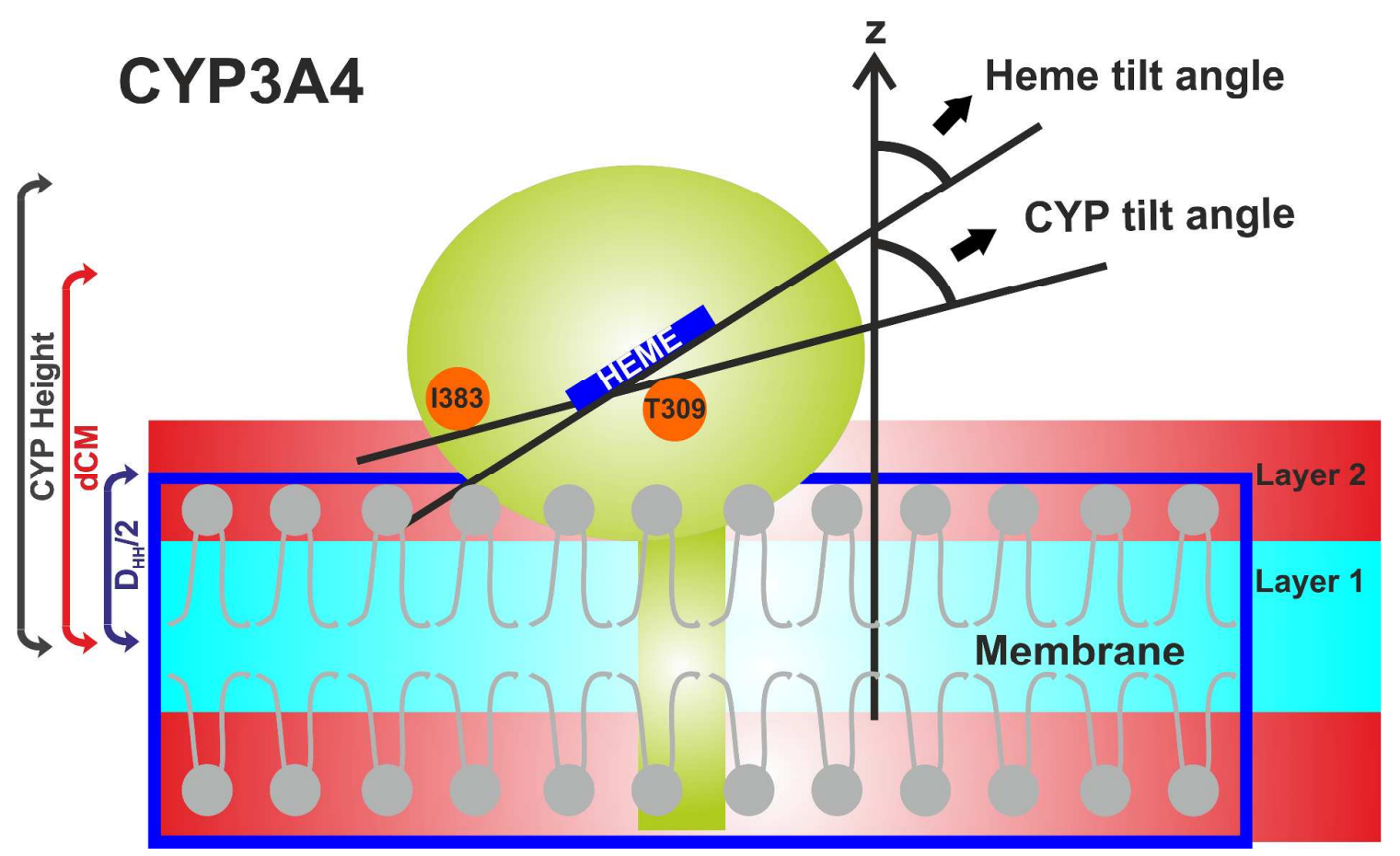

Figure S1. Schematic view of measured CYP characteristics.
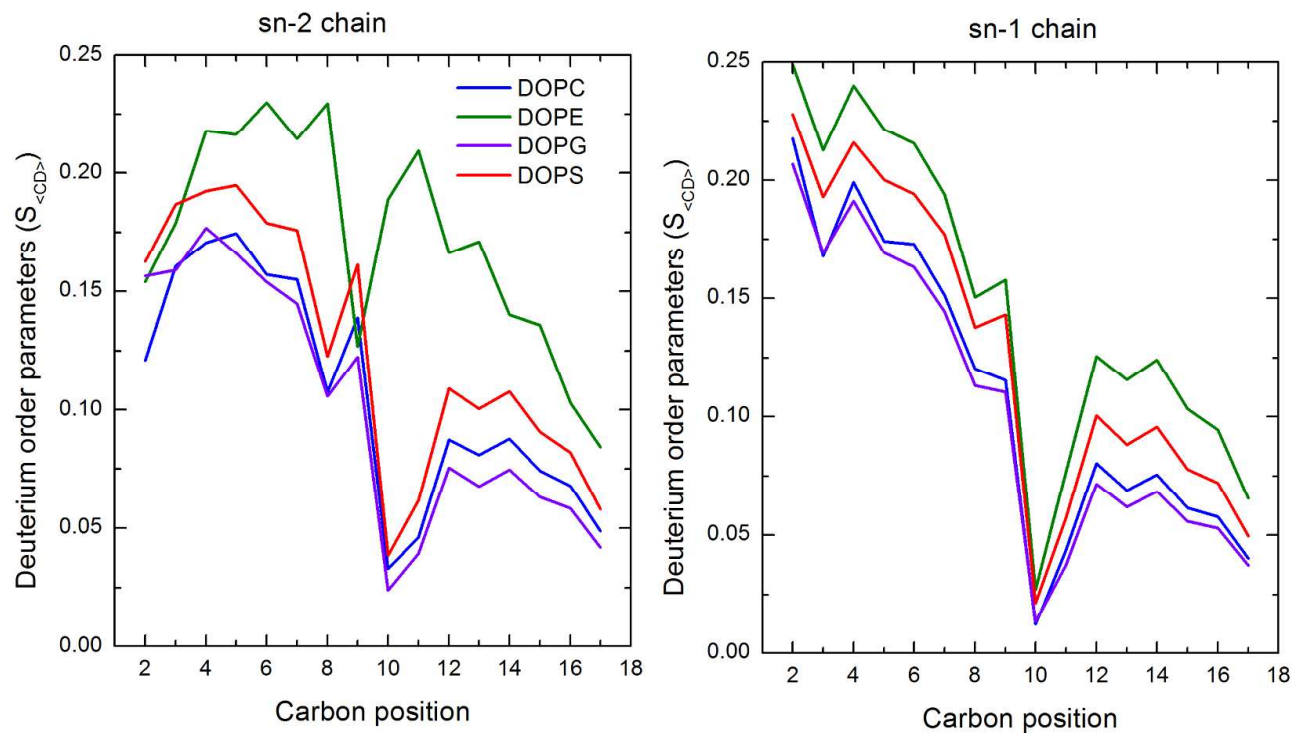

Figure S2. Average deuterium order parameters show a significant increase in the ordering of DOPE, especially in its sn-2 chain. 


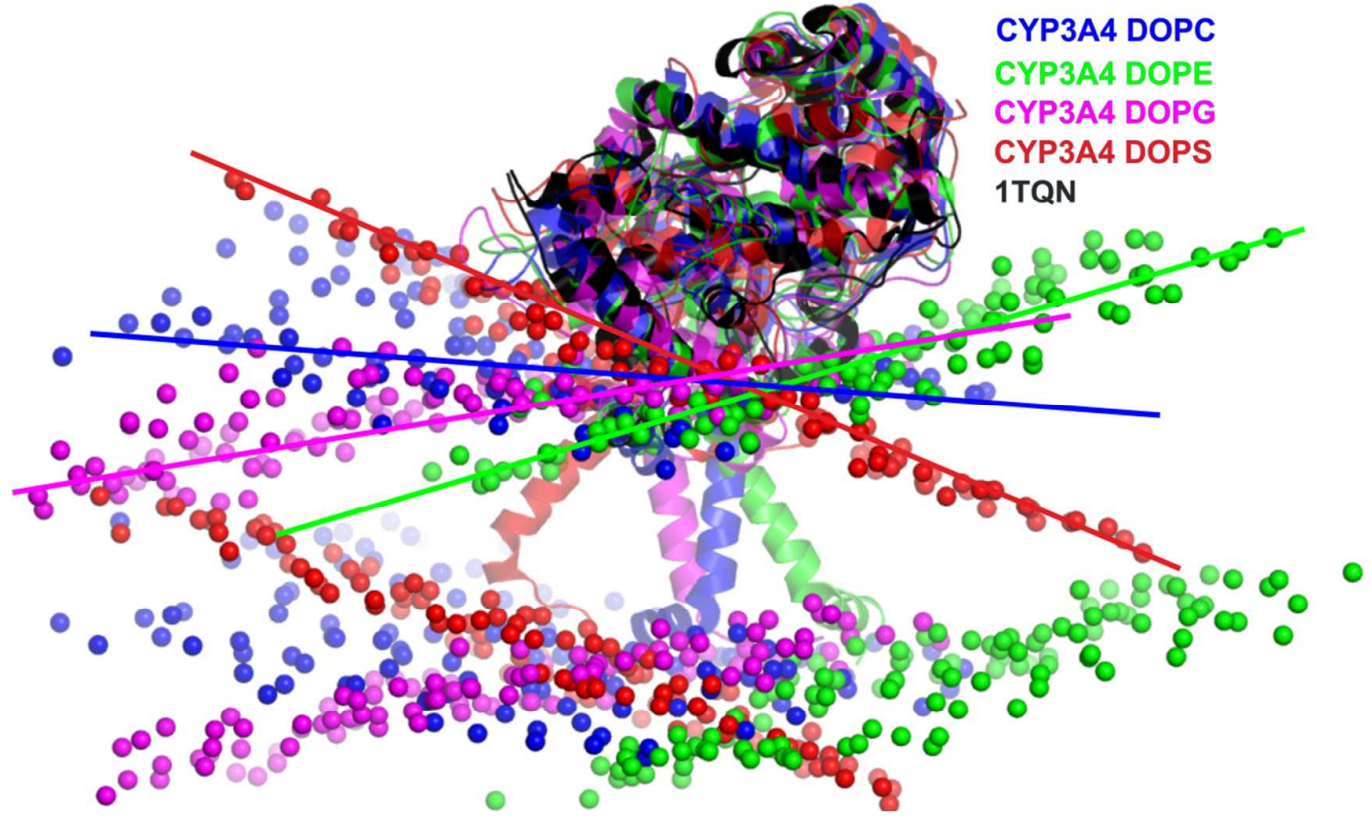

Figure S3. Comparison of crystal structure of CYP3A4 with 1000 ns DOPC, DOPE, DOPG and DOPS membranes after 1000 ns of MD simulation. The CYP3A4 is represented in cartoon and membrane phosphates as spheres and colored according to individual membrane. The crystal structure of CYP3A4 - ITQN is showed as black cartoon.

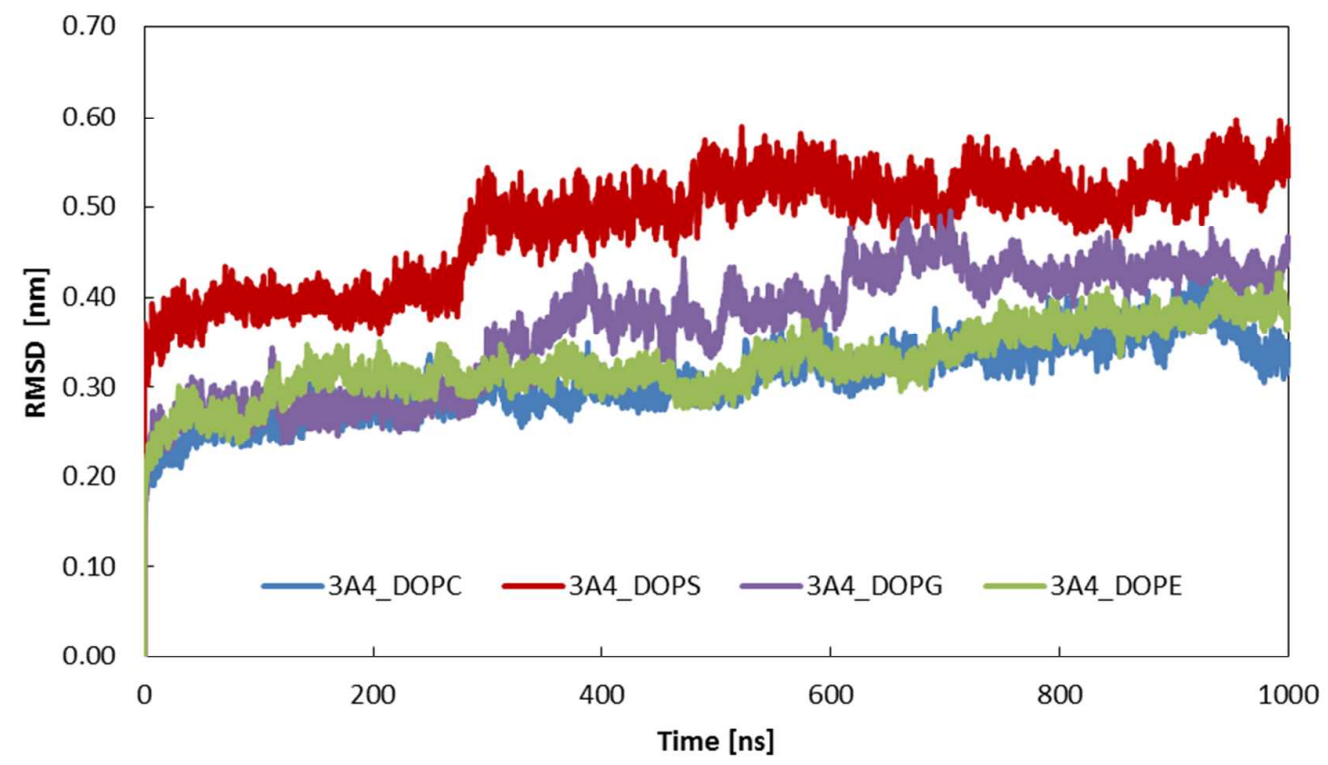

Figure S4. RMSD evolution of CYP3A4 from starting structure based on PDBID: 1TQN. 
Table S2. Average values and standard deviations of measured characteristics from whole $1000 \mathrm{~ns}$, from last 200 and last 500 ns of MD simulation.

\begin{tabular}{|c|c|c|c|c|c|c|c|c|c|}
\hline \multirow[t]{2}{*}{ Lipid } & \multicolumn{3}{|c|}{$1000 \mathrm{~ns}$} & \multicolumn{3}{|c|}{ last $200 \mathrm{~ns}$} & \multicolumn{3}{|c|}{ last $500 \mathrm{~ns}$} \\
\hline & \multicolumn{3}{|c|}{$\begin{array}{l}\text { HEME-DOPx distance } \\
{[\mathrm{nm}]}\end{array}$} & \multicolumn{3}{|c|}{$\begin{array}{l}\text { HEME-DOPx distance } \\
{[\mathrm{nm}]}\end{array}$} & \multicolumn{3}{|c|}{$\begin{array}{l}\text { HEME-DOPx distance } \\
{[\mathrm{nm}]}\end{array}$} \\
\hline DOPC & 4.14 & \pm & 0.15 & 4.24 & \pm & 0.18 & 4.20 & \pm & 0.15 \\
\hline DOPE & 4.28 & \pm & 0.25 & 4.42 & \pm & 0.13 & 4.41 & \pm & 0.14 \\
\hline DOPG & 3.64 & \pm & 0.13 & 3.61 & \pm & 0.11 & 3.66 & \pm & 0.15 \\
\hline \multirow[t]{2}{*}{ DOPS } & 3.91 & \pm & 0.14 & 3.93 & \pm & 0.07 & 3.94 & \pm & 0.10 \\
\hline & \multicolumn{3}{|c|}{$\begin{array}{l}\text { CYP-DOPx distance } \\
{[\mathrm{nm}]}\end{array}$} & \multicolumn{3}{|c|}{$\begin{array}{l}\text { CYP-DOPx distance } \\
{[\mathrm{nm}]}\end{array}$} & \multicolumn{3}{|c|}{$\begin{array}{l}\text { CYP-DOPx distance } \\
{[\mathrm{nm}]}\end{array}$} \\
\hline DOPC & 3.77 & \pm & 0.14 & 3.83 & \pm & 0.17 & 3.78 & \pm & 0.15 \\
\hline DOPE & 3.83 & \pm & 0.14 & 3.84 & \pm & 0.15 & 3.87 & \pm & 0.14 \\
\hline DOPG & 3.10 & \pm & 0.11 & 3.09 & \pm & 0.10 & 3.11 & \pm & 0.12 \\
\hline \multirow[t]{2}{*}{ DOPS } & 3.58 & \pm & 0.15 & 3.55 & \pm & 0.07 & 3.59 & \pm & 0.11 \\
\hline & \multicolumn{3}{|c|}{$\begin{array}{c}\text { F/G-DOPx distance } \\
{[\mathrm{nm}]}\end{array}$} & \multicolumn{3}{|c|}{$\begin{array}{c}\text { F/G-DOPx distance } \\
{[\mathrm{nm}]}\end{array}$} & \multicolumn{3}{|c|}{$\begin{array}{c}\text { F/G-DOPx distance } \\
{[\mathrm{nm}]}\end{array}$} \\
\hline DOPC & 2.58 & \pm & 0.18 & 2.63 & \pm & 0.49 & 2.60 & \pm & 0.19 \\
\hline DOPE & 2.73 & \pm & 0.31 & 2.85 & \pm & 0.22 & 2.88 & \pm & 0.23 \\
\hline DOPG & 1.74 & \pm & 0.16 & 1.70 & \pm & 0.13 & 1.78 & \pm & 0.19 \\
\hline \multirow[t]{2}{*}{ DOPS } & 2.15 & \pm & 0.16 & 2.05 & \pm & 0.08 & 2.12 & \pm & 0.13 \\
\hline & \multicolumn{3}{|c|}{$\begin{array}{l}\text { I-helix-DOPx distance } \\
{[\mathrm{nm}]}\end{array}$} & \multicolumn{3}{|c|}{$\begin{array}{c}\text { I-helix-DOPx distance } \\
{[\mathrm{nm}]}\end{array}$} & \multicolumn{3}{|c|}{$\begin{array}{c}\text { I-helix-DOPx distance } \\
{[\mathrm{nm}]}\end{array}$} \\
\hline DOPC & 3.82 & \pm & 0.16 & 3.87 & \pm & 0.21 & 3.83 & \pm & 0.17 \\
\hline DOPE & 3.92 & \pm & 0.25 & 3.98 & \pm & 0.19 & 4.02 & \pm & 0.19 \\
\hline DOPG & 3.06 & \pm & 0.15 & 3.02 & \pm & 0.13 & 3.08 & \pm & 0.18 \\
\hline \multirow[t]{2}{*}{ DOPS } & 3.57 & \pm & 0.18 & 3.57 & \pm & 0.08 & 3.59 & \pm & 0.12 \\
\hline & \multicolumn{3}{|c|}{$\begin{array}{c}\text { HTA* } \\
{\left[{ }^{\circ}\right]}\end{array}$} & \multicolumn{3}{|c|}{$\begin{array}{l}\text { HTA* } \\
{\left[{ }^{\circ}\right]}\end{array}$} & \multicolumn{3}{|c|}{$\begin{array}{l}\text { HTA* } \\
{\left[{ }^{\circ}\right]}\end{array}$} \\
\hline DOPC & 59.10 & \pm & 7.74 & 64.77 & \pm & 5.57 & 63.65 & \pm & 6.09 \\
\hline DOPE & 64.71 & \pm & 13.31 & 75.19 & \pm & 5.27 & 73.21 & \pm & 5.56 \\
\hline DOPG & 77.52 & \pm & 4.86 & 75.41 & \pm & 4.91 & 77.32 & \pm & 5.00 \\
\hline DOPS & 72.49 & \pm & 6.36 & 72.68 & \pm & 5.67 & 68.59 & \pm & 5.20 \\
\hline
\end{tabular}

*HTA - Heme tilt angle 


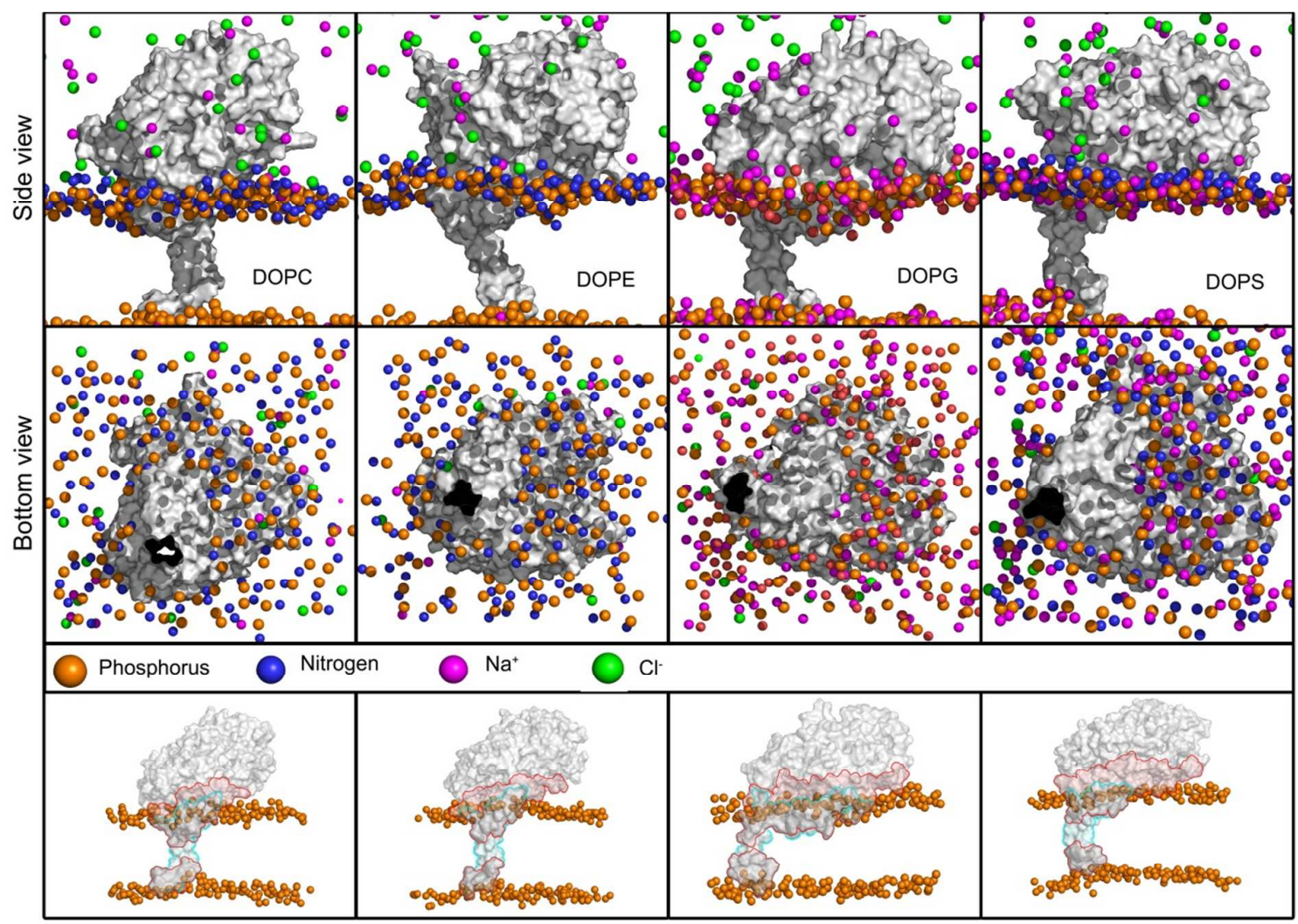

Figure S5. Side view and bottom view (from the membrane) at CYP3A4 with displayed ions (magenta and green balls). In case of charged DOPG and DOPS, $\mathrm{Na}^{+}$ions together with charged head groups of lipids form a unique environment in contact with CYP $3 A 4$ (gray surface).

The lowest panel shows the distribution of amino acids in contact with membrane (see Table 1 in main text) in contact with lipid tails (cyan, L1) and with lipid head groups (red, L2). 


\section{CYP3A4}

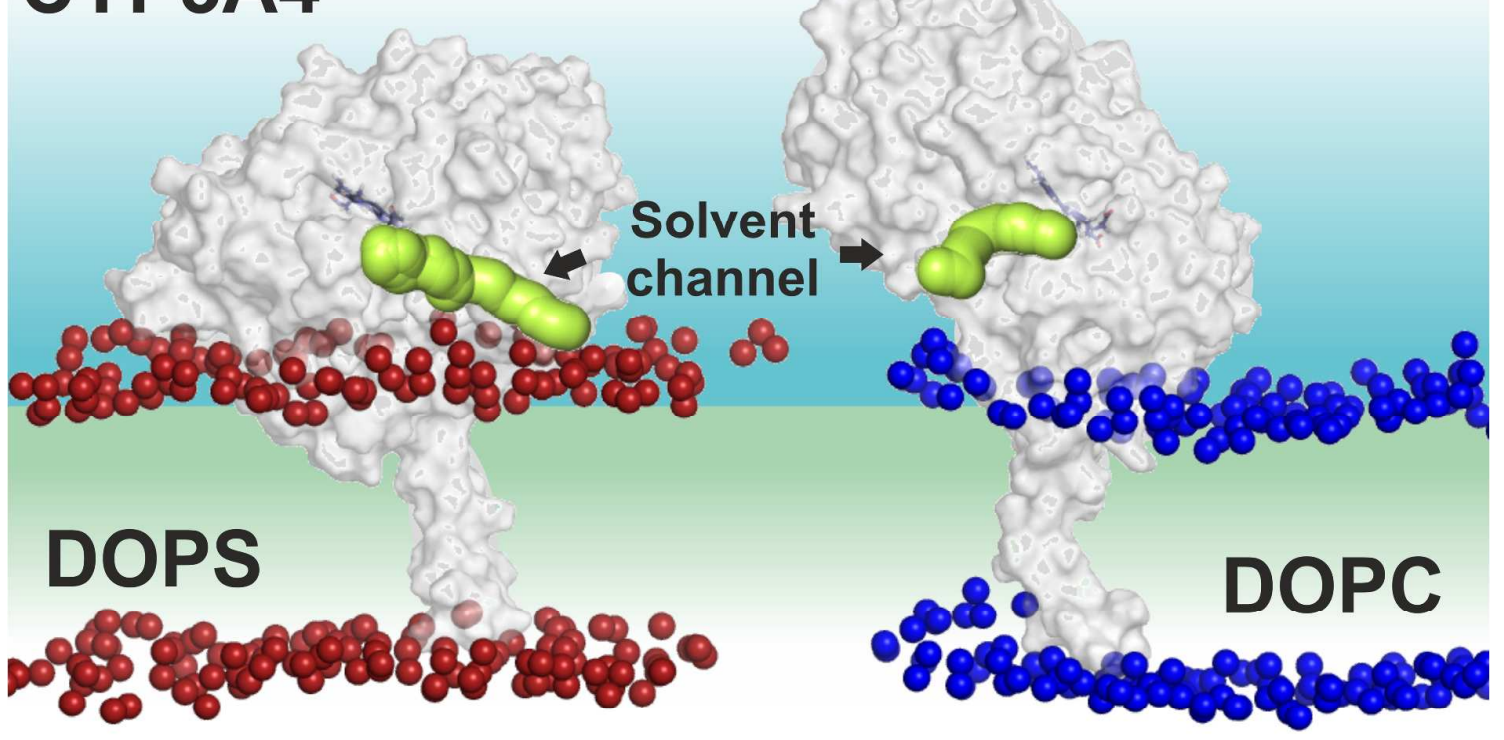

Figure S6. Scheme of changes of channel mouths positions in DOPC and DOPS membranes. The changes in the tilt and immersion depth cause a significant difference in channel mouth position that may cause a change in catalytic activity.

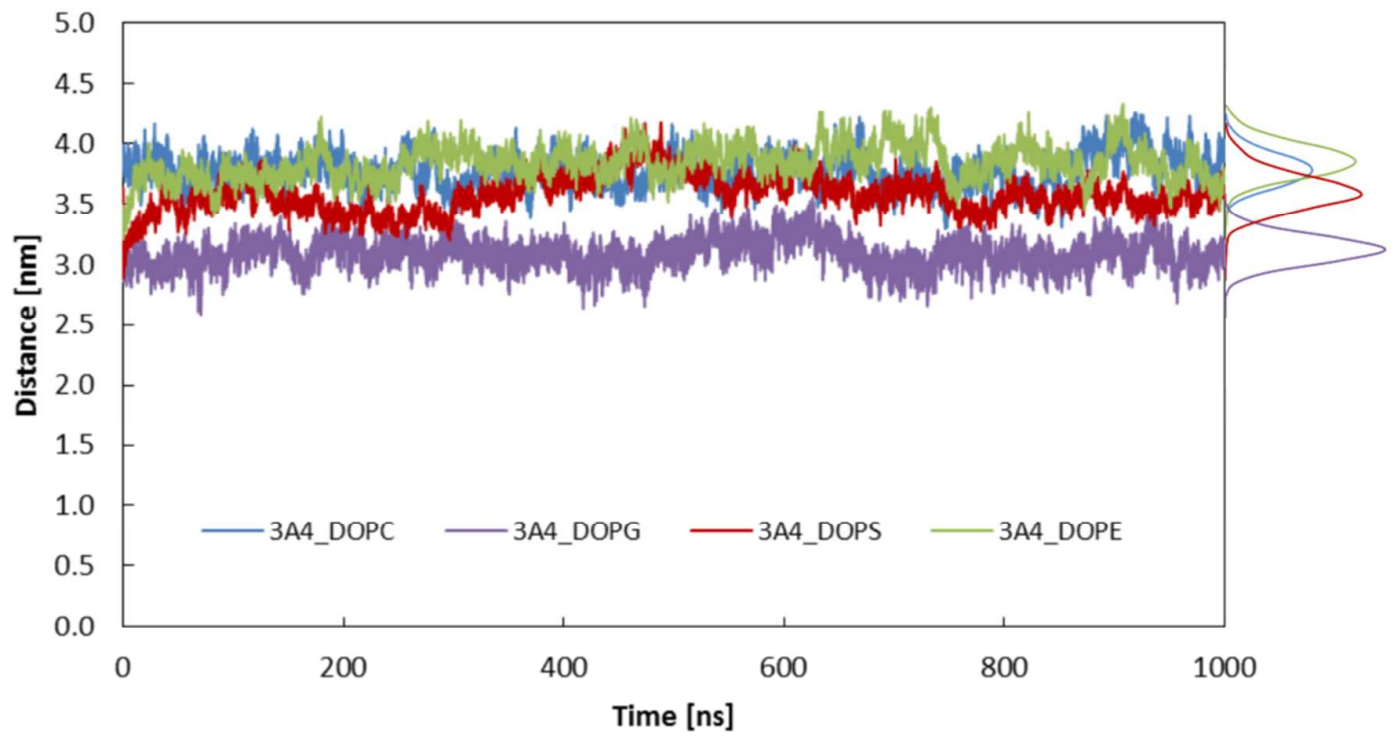

Figure S7. Distance of CYP3A4 and respective DOPx membrane of 1000 ns long MD simulation (which describes depth of immersion of CYP3A4 in the membrane). Lipids are colored as follows: DOPC - blue, DOPE - green, DOPS - red, DOPG - purple.) The right panel shows histogram depicted occurrence of respective distance during the $1000 \mathrm{~ns}$ of MD simulation. 


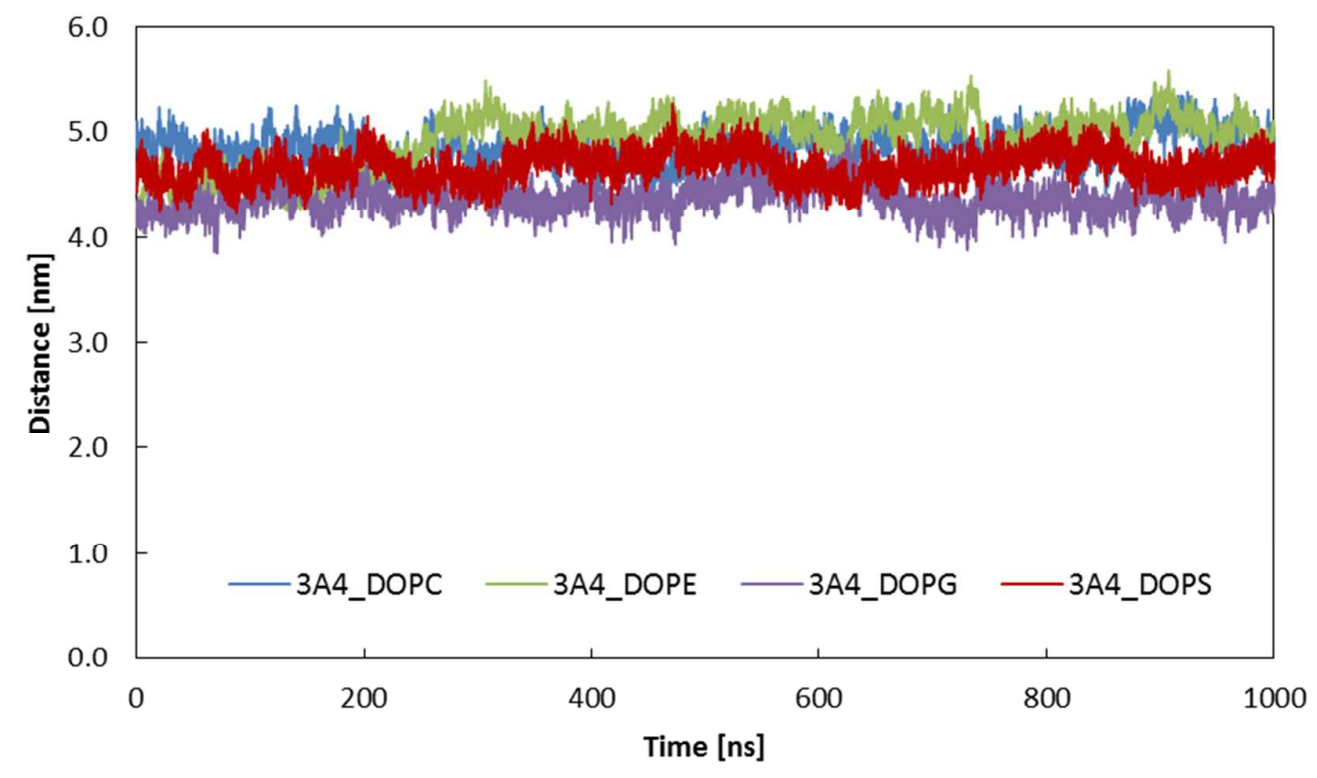

Figure S8. Distance between CPR contact amino acids (441, 442, 445 and 446) and center of mass of the membrane during $1000 \mathrm{~ns}$ long MD simulation. Lipids are colored as follows: DOPC-blue, DOPE - green, DOPS - red, DOPG - purple).

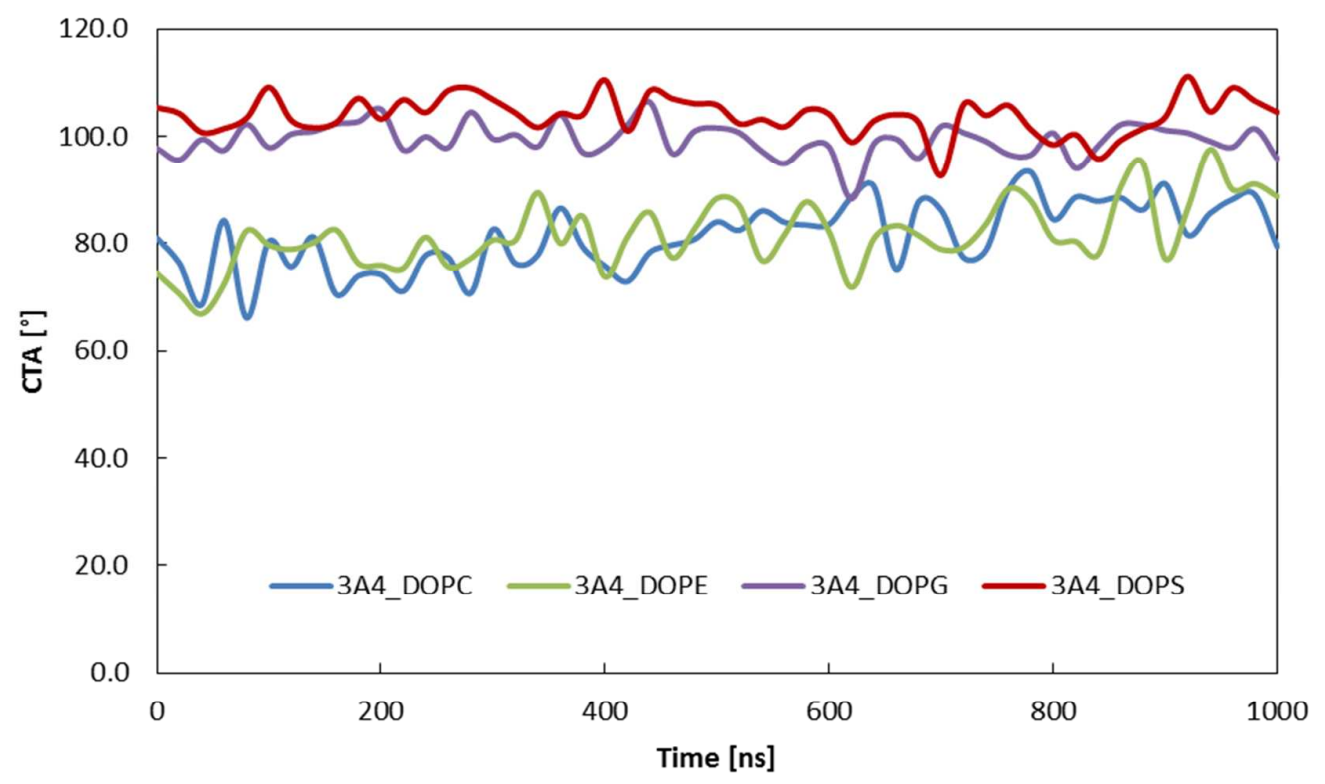

Figure S9. CYP tilt angle (CTA), shows the fluctuations of CYP3A4 orientations on the membrane. CTA is defined by angle between middle of I helix and B1-sheet and a z-axis (between highly conserved threonine T309 on I-helix and I383 on the B1-sheet. The PC and PE membranes behave similar as well as DOPG and DOPS membranes show similar trends). 

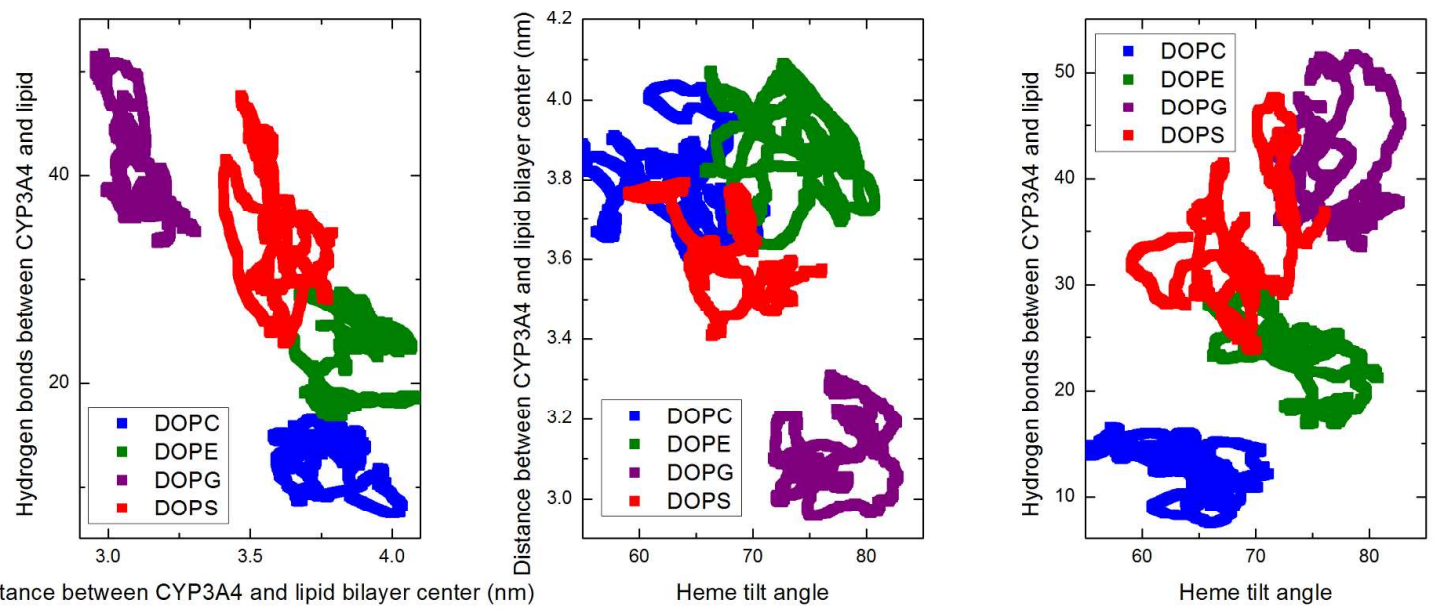

Figure S10. Correlation of monitored properties during the simulation. Higher number of hydrogen bonds is connected with a lower distance of center of masses of CYP3A4 and the membrane (left panel) and with a higher heme tilt angle (right panel). However, the heme tilt angle and the distance of CYP $3 A 4$ and the membrane are not too correlated (middle panel).

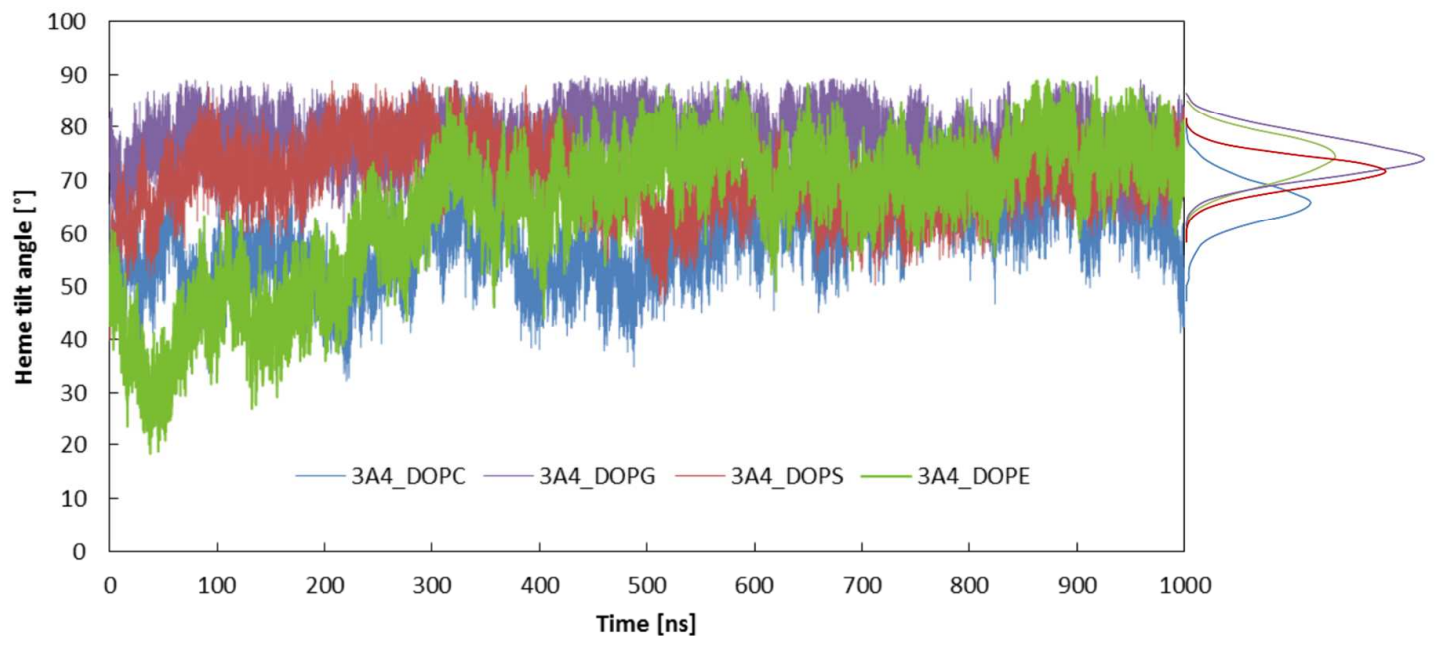

Figure S11. Heme tilt angle of CYP3A4 on different membranes (DOPC-blue, DOPE - green, DOPG - purple, DOPS - red), whole 1000 ns of MD simulation. The right panel shows histogram depicts occurrence of respective heme tilt angle during the $1000 \mathrm{~ns}$ of MD simulation. 


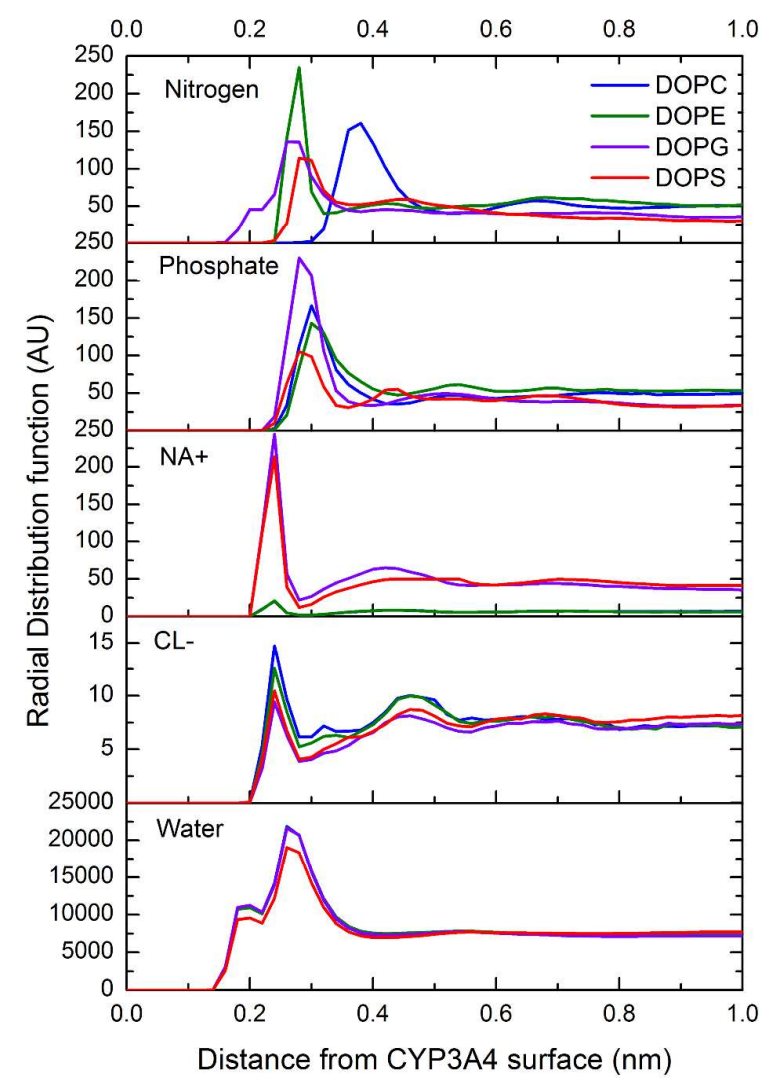

Figure S12. Radial distribution functions of terminal nitrogens (in case of DOPG a terminal oxygen), phosphorus atoms, ions and water around a surface of CYP $3 A 4$. 


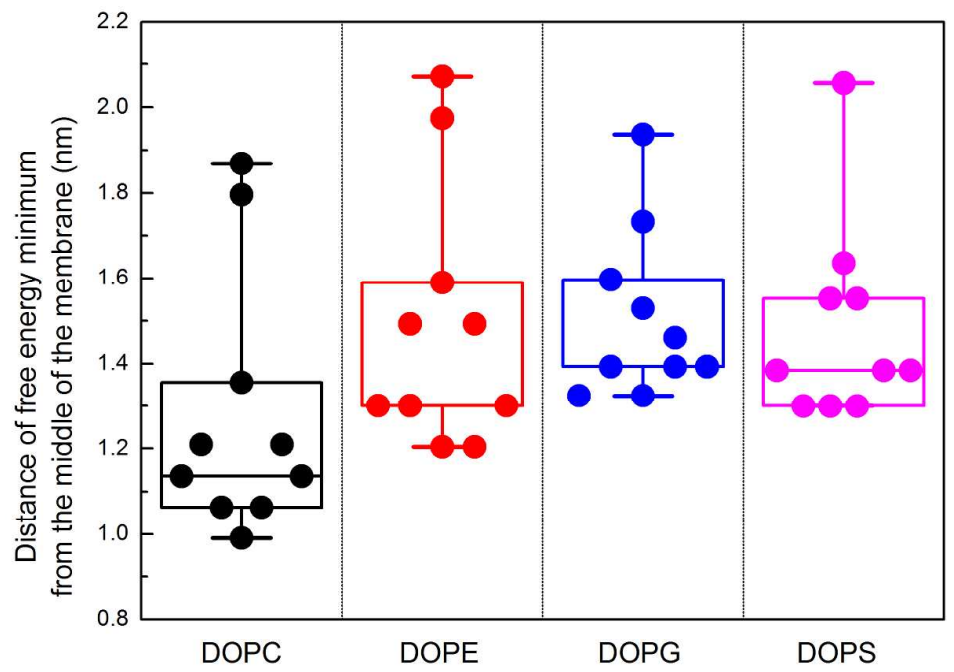

Figure S13. Box-and-Whiskers plot of distances of free energy minima of substrates and metabolites of CYP3A4 in respective membranes as a result of COSMOmic calculation. The circles show the position of individual molecules, boxes show a distribution from 25 to 75 percentiles.

Free energy profiles for several substrates and metabolites of CYP3A4 were calculated by COSMOmic software ${ }^{37}$ from the COSMOtherm 15 package. The MD simulation of pure lipid membranes were used for the atom distribution calculation and 10 lipid conformers were considered as recommended earlier. ${ }^{17,41}$ The geometries and $\sigma$-profiles of DOPx, water and guest molecules were obtained by DFT/COSMO calculations at the BP/TZVPD-fine level of theory. ${ }^{67,68} \mathrm{~A}$ single conformation as a result of geometry optimization was used. Following molecules were used: acetaminophen, acetimidoquinone (NAPQI), diazepam, temazepam, oxazepam, warfarin, 10-hydroxywarfarin, nifedipine, N-hydroxyarginine, theophylline, and 3methylxanthin. Free energy profiles were calculated at $310 \mathrm{~K}$. The bilayers were separated into 50 layers, a total of 162 orientations of the solute molecules were used for each lipid conformer representation to produce individual free energy profiles. The final free energy profile was averaged over the individual free energy profiles of all lipid conformers. 
Table S3. Preferences of CYP membrane domains based on the charge for important mammalian CYPs. Detailed analysis: Exterior amino acids were selected as exposed amino acids with exposed area of at least $3 A^{2}$ using FindSurfaceResidues.py script from Pymol Scripts repository. (https://github.com/PymolScripts/Pymol-script-repo/raw/master/findSurfaceResidues.py). Distal side was selected as a side above plane of heme (including heme). Due to its pKa we do not take into account histidines.

\begin{tabular}{|c|c|c|c|c|c|c|c|c|c|c|c|c|c|c|}
\hline \multirow{3}{*}{$\begin{array}{l} \\
\text { CYP } \\
\end{array}$} & \multirow[b]{3}{*}{ total } & \multirow[b]{3}{*}{ distal } & \multirow[b]{3}{*}{ proximal } & \multirow[b]{3}{*}{ TM helix } & \multirow[b]{3}{*}{ PDBID } & \multirow[b]{3}{*}{ (Dis)Order } & \multirow{2}{*}{\multicolumn{2}{|c|}{$\begin{array}{c}\text { \# of positive } \\
\text { surface AA } \\
\text { (Arg + Lys) }\end{array}$}} & \multirow{2}{*}{\multicolumn{2}{|c|}{$\begin{array}{c}\text { \# of negative } \\
\text { surface AA } \\
(\text { Asp + Glu) }\end{array}$}} & \multicolumn{4}{|c|}{ Total \# of charged AA } \\
\hline & & & & & & & & & & & \multicolumn{2}{|c|}{ Surface } & \multicolumn{2}{|c|}{ Total } \\
\hline & & & & & & & distal & proximal & distal & proximal & distal & proximal & distal & proximal \\
\hline rabbit $1 \mathrm{~A} 2$ & 12 & N/A & N/A & N/A & N/A & $\begin{array}{l}\text { order }^{21,22,24,25} \\
\text { anionic rich }^{60}\end{array}$ & & & & & & & & \\
\hline human $1 \mathrm{A2}$ & 10 & 2 & 7 & 1 & 2hi4 & & 32 & 24 & 30 & 17 & 62 & 41 & 64 & 41 \\
\hline human $2 \mathrm{C} 8$ & 9 & 10 & -4 & 3 & $2 \mathrm{nnj}$ & & 33 & 21 & 19 & 25 & 52 & 46 & 56 & 50 \\
\hline rabbit 2B4 & 6 & 6 & -1 & 1 & 1 po5 & dis + order $^{20}$ & 36 & 19 & 27 & 19 & 63 & 38 & 68 & 39 \\
\hline human 1A1 & 5 & 4 & -1 & 2 & $4 \mathrm{i} 8 \mathrm{v}$ & & 26 & 24 & 19 & 25 & 45 & 49 & 48 & 51 \\
\hline rabbit 1A1 & 3 & $\mathrm{~N} / \mathrm{A}$ & N/A & $\mathrm{N} / \mathrm{A}$ & $\mathrm{N} / \mathrm{A}$ & $\operatorname{dis}^{20,21,25}$ & & & & & & & & \\
\hline rabbit 2E1 & 3 & N/A & N/A & N/A & N/A & $\operatorname{dis}^{20}$ & & & & & & & & \\
\hline human $2 \mathrm{C} 9$ & 3 & 4 & -1 & 0 & $1 \mathrm{r} 90$ & & 30 & 24 & 21 & 25 & 51 & 49 & 56 & 51 \\
\hline human 2B6 & 3 & 5 & -2 & 0 & 3 ua5 & & 36 & 18 & 28 & 19 & 64 & 37 & 69 & 38 \\
\hline human 2E1 & 3 & 3 & 0 & 0 & $3 \mathrm{koh}$ & & 35 & 23 & 28 & 23 & 63 & 46 & 67 & 48 \\
\hline human 3A4 & 3 & 6 & 0 & -3 & 1 tqn & & 39 & 17 & 34 & 16 & 73 & 33 & 78 & 34 \\
\hline human $2 \mathrm{C} 19$ & 0 & -2 & 1 & 1 & $4 \mathrm{gqs}$ & & 28 & 24 & 27 & 23 & 55 & 47 & 60 & 49 \\
\hline human 2D6 & -2 & 1 & -5 & 2 & $3 q \mathrm{qm} 4$ & & 32 & 14 & 28 & 18 & 60 & 32 & 65 & 33 \\
\hline
\end{tabular}

